\title{
Kontaktallergien: Nickel weiterhin relevant
}

— Allergische Kontaktekzeme kommen bei Kindern und Jugendlichen häufig vor (5-20\%). Nickelexpositionen spielen dabei nach wie vor eine wichtige Rolle. So kann etwa stundenlanges Telefonieren mit dem Handy Ekzeme auf der Wange hervorrufen. Als Ursache fand man eine Nickelfreisetzung über $0,5 \mu \mathrm{g} / \mathrm{cm}^{2}$ pro Woche. Klassischer Auslöser von Kontaktallergien sind außerdem Duftstoffe oder Gummiinhaltsstoffe. Doch auch topische Medikamente wie Neomycin oder Bufaxamac stehen laut Prof. Peter Thomas, München, auf der „Hitliste“, wie er in seinem Vortrag auf dem 15. Biedersteiner Kongress berichtete. Seit einigen Jahren gesellen sich auch noch „Trendallergiestoffe“, wie etwa der schwarze Farbstoff (Paraphenylendiamin) dazu, der bei temporären Tatoos verwendet wird.
„Besteht Verdacht auf Kontaktallergie, lohnt sich auf jeden Fall ein Standardepikutantest", erklärte Thomas. Dieser sei auch bei 5-6-Jährigen erlaubt. Allerdings mit einer verkürzten Substanzpalette und indem man das Pflaster bereits nach 24 Stunden entfernt.

Kinder und Jugendliche haben über Modeschmuck häufig Kontakt mit potenziell allergieauslösenden Metallen. Denn trotz der europäischen Nickeldirektive „ist das Thema Nickel weiterhin relevant", betonte Thomas. So enthält zum Beispiel ein Teil des Billigschmucks nach wie vor hohe Nickelanteile: etwa Kinderhaarspangen (79,4\%), Kinderfingerringe (20\%), Ohrringe (14,5\%) [Sci Total Environ 2009; 401: 5315-8].

Ein weiteres Risiko für Metallallergien entsteht durch Piercings. „Auffällig ist, dass etwa die Hälfte der Personen beim

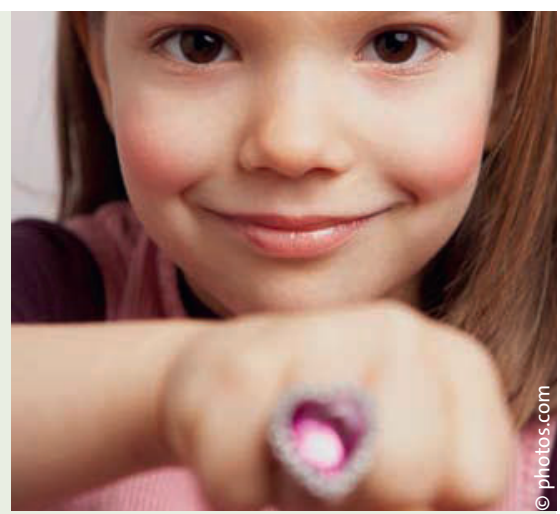

Billigschmuck enthält trotz des europaweiten Verbots häufig noch noch Nickel.

Setzen des Piercings jünger als 18 Jahre ist", berichtete Thomas. Auch gaben 18\% der Befragten einer deutschen Studie temporäre Entzündungen an [Eur J Dermatol 2008; 18: 26-8].

mha

Vortrag: Metallallergie - ein Thema im Kindesalter? 